\title{
Zen shiatsu - the japanese way of acupuncture without needles
}

\author{
Volume 6 Issue 3 - 2017 \\ Theodoros Haralabidis \\ Spa manager, Certified massage therapist, Oriental Medicine \\ Training Centre, Greece
}

\section{Opinion}

Shiatsu is an ancient non-invasive therapeutic technique, a comprehensive treatment system based on the same concepts and roots with Chinese Acupuncture and Traditional Chinese Medicine (TCM).

According to this theory, the human body and its internal organs function with the power and influence of Chi Energy.

The imbalance between our mental, physical, emotional and spiritual world, is the reason that our body gets sick. What Shiatsu does is, to restore this balance of energy in the body and bring a harmony between these aspects of our life.

Shiatsu is performed by finger pressure (instead of needles) on the acupoints.

\section{How does shiatsu work?}

Shiatsu was developed in Japan by combining a Chinese type of massage, the Anma and Western physical therapy techniques. The Shiatsu techniques include stretching, grip and inclination of therapist's body weight over many parts of the recipient's body. The objective is to improve:
i. The energy flow
ii. The blood circulation
iii. The flexibility and
iv. The Posture of the body Pressure and contact is applied through
v. Hands
vi. Thumbs
vii. Fingers

viii. Forearms

ix. Knees And

$\mathrm{x}$. Feet

It is performed with the recipient in various positions such as supine, side, prone, sitting. The treatment focuses mainly along specific channels ("Meridians") from where the body Chi energy passes through. Although Shiatsu literally means 'finger pressure', the soul of Shiatsu is communication through touch. Shiatsu which meets the needs of the recipient cannot be achieved only with technical skills and intellectual study. So that it will be effective, requires sensitivity so that we feel and influence positively the quality of energy that exists within the recipient's body.

\section{Shiatsu for common ailments}

Shiatsu cannot be used for treating diseases, as for such cases we must reach out to our doctor. However, what it can offer is

i. To relieve from some symptoms,

ii. To prevent and minimize some undesired evolution and

iii. To complement the treatment of some specific health professionals (if permitted)

iv. Usually, some of such discomfort cases are

v. Mental and Physical fatigue,

vi. Stress

vii. Worry,

viii. Insomnia,

ix. Back pain and sciatica,

$\mathrm{x}$. Period pains, as it can assist on strengthening the immune system as well.

\section{How is it performed?}

Shiatsu is done with the recipient wearing light and comfortable cotton clothes, usually lying on a comfortable cotton or futon mattress on the floor. Usually, an experienced therapist will use some pillows under the recipient's feet or hands, to cover the gap and absorb any possible mechanical restoration and traction during the pressure.

The position can be supine, prone, lateral or sitting. There is continuous contact between the recipient and the Shiatsu therapist, while the first relaxes and the second maintains continuous physical, 
energy, and emotional communication in a relaxed meditative atmosphere. Do not forget that this is an energy healing technique!

Thumbs, elbows, fingers, forearms, feet pressure is used by the therapist. Also, some stretches from the Western Physio or Oriental Yoga!

\section{When it can be performed?}

The fact that there is no need for the recipient to take off his/her clothes, like they do in a Deep Tissue massage, Shiatsu is suitable in any place or in any time of the day.

Provided there is appropriate peaceful atmosphere without noises that distract our attention. No special equipment is required such as massage oils, only the expert hands of the therapist.

\section{When is not suitable to do shiatsu}

Generally Shiatsu is a very safe technique. Here it holds the same with the other massage treatments. However, we would avoid just to press on some points or locally on some specific areas. Such points are, four points which may cause miscarriage to a pregnant woman or undesired premature birth and some points contraindicated in some people with hypertension or hypotension. Also, we never press points with inflammation, edema, trauma, varicose veins or pimples.

An experienced professional therapist will first take a short medical history from the client and then will go through the Shiatsu session.

\section{Traditional Chinese medicine - some basic terms}

One to understand how Shiatsu works, first should consider the core of Traditional Chinese Medicine (TCM), in other words how this ancient Chinese system treats the disease. In Western Medicine, a doctor will deal more with the effects of the disease and will try to minimize or eliminate them completely.

In TCM, the therapist will make a completely different diagnosis. $\mathrm{He} / \mathrm{she}$ will observe the whole picture of the patient, the overall external appearance, the way the patient moves, speaks and behaves, until the color of the patient's urine, the tastes that prefers and his/her emotional state. The therapist will try to build a profile of the patient, which will consist of some "Syndromes disharmony."

The Oriental therapist, the Acupuncturist or the Shiatsu therapist is expected to treat this Disharmony and to restore it to its original equilibrium (state).

\section{The energy chi concept}

Chi is the vital force that supports every activity of the human body, mind and spirit. According to the Chinese theory of Taoism, Chi is everywhere in the universe surrounding us. It is in the air we breathe, the food we eat, inside us as a legacy of our parents.

The Chinese character for Chi suggests that it is something which, at the same time is material and intangible Steam, gas \# (uncooked) rice $=\mathrm{Chi}$

This clearly shows that Chi can be as intangible as steam and so dense material as rice. It also shows that, Chi is a thin element (steam) coming from something concentrated (rice), such as steam produced when the rice is cooked.

Chi has been translated like many concepts, such are: Energy, Material Force, Matter, Ether, Material Energy, Life Force, Vital Force, Vital Dynamic, and Moving Energy.
The inability to be translated exactly stems from its nature of taking various forms of expression. Chi nourishes the vital organs of the human body, the Blood and other Body Fluids and our Mind. In Traditional Chinese Medicine bibliography you will see the word blood written with a capital B. This is to distinguish the difference between the red fluid of blood that runs in our veins from the Blood, one of the shapes Chi gets and one of the Vital Forces.

It is also responsible for all the processes of life, from conception, childbirth, to growth and death. "Chi produces the human body, exactly like the water becomes ice. As the water freezes (and becomes ice) so Chi coagulates to form the human body. When the ice melts is water. When a person dies, becomes spirit (Shen) again. It is called spirit, just like molten ice is changing its name to water. 'Wag Chong (Chinese philosopher during the Han Dynasty, AD 27-97).

\section{The elements yin \&yang}

It is the oldest $(700 \mathrm{BC})$ and significant philosophical theory in Chinese medicine, which is completely different from any Western. Therefore, according to this theory, everything in life, every phenomenon can be exactly what it is, and also it's opposite. Also, the Yin element contains within it the seed of Yang and the Yang Element contains within it the seed of Yin Element.

Each element complements and supports each other. They are two phases in a circular motion. For example, the day alternates to night progressively, there is time of day that contains little dark and there is time of night that contains light in it. Like the Five Elements, so YinYang have some specific characteristics, qualities, properties. Some of them are Light-Dark, Motion-Rest, Material and Intangible, EnergyMatter, Water-Fire, etc.

Yin-Yang keeps under its power and controls some internal organs. Between them there are some principles of co-existence and relationship, which when they are violated a disharmony and imbalance is occurred to human organs and body.

\section{The 5 elements}

This is an ancient Theory (1000 -770 BC), that together with the Yin-Yang theory they are the basis of Traditional Chinese Medicine. The term has been used by ancient Greek philosophers, Air-FireEarth-Water, roots, elements, primordial bodies, etc. In fact, it is about five processes, five qualities; five stages of a cycle or alternating phenomena. The 5 Elements are Wood, Fire, Earth, Metal and Water.

Each element has specific characteristics, qualities, properties that in Shiatsu practice are used in Physiology, in Pathology, in the diagnosis and recovery strategy that we will pursue.

Some of these are the flavors, sounds, odors, some senses, climates, etc. Each item is under the influence of an internal vital organ such as the Liver, Heart, Spleen, Large Intestine, Kidney, Gall Blader, etc. Each organ influences, governs, controls, affects another and is controlled and affected by someone else. There is a regular balanced cycle of control of all previous procedures, but there is also an imbalanced cycle and one insulting cycle.

As is mentioned above, the therapist who uses T.C.M, the Acupuncturist or the Shiatsu therapist is called to restore this Disharmony and to restore it to its original equilibrium (state).

\section{The meridians-what is tsubo?}

According to Chinese Medicine, energy is released in some channels on the surface of our body. The Meridians are the pathways 
for the circulation of Chi and present in everyone's body. 12 Meridians have been identified and every one of them takes its name from the specified internal organ passes through or is connected eg Heart Meridian, Stomach Meridian, Bladder Channel, etc.

Over the Channels there are specific points, on which the Shiatsu practioners puts pressure or the acupuncturist's needles. The points are numerous and located on or near veins, arteries, tendons, nerves. And no, I am afraid they are not the points of the lymphatic system, as some health professional's claim, rationalizing the system of Traditional Chinese Medicine with Western mindset of conventional medicine!

The points in Japanese are called Tsubo and these in turn have names. In Chinese, for some of the points are used somewhat poetic names such as "Gate of Life/Mingmen", for some others quite illustrative names as "Intersection of 3 Yin / Sanyinjiao". In English we use the name of the Channel belonging point numbered, such as Du -4 and Spleen-6 respectively for the previous two.

Each point has specific functions, techniques by which is pressed, actions that it does, and sometimes contraindications. So, one point can strengthen an internal organ or Yin or Yang and other point can calm or descent the Chi. Some enhance the (birth) delivery, so if you do not want to have unexpected results, do not press it! A well, efficiently and professionally educated therapist of Shiatsu, in order to identify the points should certainly have learned first Anatomy, Physiology and Pathology and point location!

\section{The kyo/jitsu concept}

In the system of Zen Shiatsu, whose founder was the Japanese teacher of Shiatsu Shizuto Masunaga (1925-1981), there are the concepts Kyo/Jitsu. They have been translated as Empty/Full, ie deficient and excess Chi respectively, referring to a tsubo point The Jitsu/Full points are easily identifiable by touch (and by the kin eye sometimes), as they would usually are protruding and aching, eg in a back. The Kyo/Empty points are identified with difficulty and usually they are deeper in the body. The Shiatsu therapist should have enough experience to distinguish the difference, and to turn them into their original balance effectively. This is the difference of a good shiatsu session with a very good session Shiatsu!

The teacher of Zen Shiatsu, Chris Jarmey whose courses I had the fortune and honor to attend many times, in his book - 'Shiatsu, The complete guide' (with G. Mojey and my greek teacher Ilaira Bouratinou) likens the Kyo/Jitsu like a house which someone is banging the door. Depending ie, if someone is inside the house and not or someone can be inside but does not wishes any visitors or the opposite, someone welcomes you and shows you he was expecting you to knock the door!

\section{The vital substances concept}

As Vital substances in TCM is meant Qi, Blood, the Essence and the Body Fluids. That is, another powerful system of important functions. About Chi we discussed above. What we have not said is that Chi protects, nourishes, warms our bodies, transforms and transports food and our blood, and also holds and uplifts. With the term Blood (with a capital A) we do are not just referring to our blood, but a form of Chi, more dense and material (ie Yin). Blood is the "Mother of Chi, it nourishes the body and the mind and cools down the internal organs. The deficiency of blood cause imbalances mainly in Mind.

'Jing' Essence is a fine term of TCM, which one cannot find an equal term in Western Medicine and its meaning is hard to explain!
The human body takes energy from the air we breathe, the food we eat and from a third source, the Essence. What we mean by Essence is, imagine a substance which exists within us inherited from our parents! This substance that nourishes the fetus inherited from our parents at conception. It affects our development, our reproduction and sexual maturation, and in general what we call our "constitution" among some other things. It's what makes each of us unique!

The Essence is unique and is replenished very to not at all. According to some specialists Yoga, Tai Chi/Qi Gong, Asian Martial arts, etc. help to be replenished. Imagine it as some money left for you by your parents to a Bank account, to draw in a case of an emergency! Say, for hard times ... By Body Fluids we mean sweat, saliva, semen, the mucus, lymph, etc. The original source is the food and our drink. Their sudden or prolonged deficiency can lead to the immediate lack of blood eg Sauna or the emesis (deliberate vomiting) yogic techniques. Their accumulation causes swelling.

\section{The functions of the internal organs}

This is about another system of the Traditional Chinese Medicine Theory, according to which the functions of internal vital organs control, store and affect the Vital Substances. In Western Medicine internal organs mostly have an anatomical role, while in Chinese Medicine besides their anatomical role, possess some relationships with our feelings, body tissues, sensory organs, mental strength, etc. For example, the Heart governs Blood, is related to Joy and manifested in facial complexion, while the Liver controls the menstrual cycle, is associated with anger and manifests in nails, etc.

\section{The causes of disease}

Traditional Chinese Medicine sets balance as the key to health. Balance in work out exercise, at rest, in our diet, in our sexual activity, climate and more. When prolonged period overturned the balance occurs disharmony. The disharmony is manifested in weakness. According to Chinese medicine, diseases may have to do with our inner world or with the external pathogenic conditions in which we live or just to other causes.

Internal causes: may be our emotions such as fear, joy, sadness, shock, excessive thinking, worry, anger. Each one of these emotions infects the Qi affecting a specific organ, eg Worry affects the Spleen, Grief affects the Lungs, etc.

External causes: may be the climatic factors such as cold, heat/fire, the summer heat, dampness, dryness and wind. The Chinese Medicine here again uses a poetic term and refers to them as "The 6 Demons" because they try to intrude inside the human like Demons!

Other causes of disease are a poor constitution/heredity, overexertion in physical/mental work and exercise, excessive sexual activity, poor dietary hobbits, trauma, parasites \& poisoning, incorrect treatment.

Sexual activity for example, according to the book "Classic of the simple girl" (Dynasty Sui 581-618), must be exercised in accordance with the age of the man or the woman and the average state of health. eg for men 30years old every day, 40years old every 3days, 50years old every 5days, etc. Of course this should not be taken for granted, but only as a larger point of view!

Let's stay a little longer in males. According to Chinese Medicine, male ejaculation has a relationship with the loss of Essence which is housed at Kidneys. On the contrary, women have less Essence loss, as it has to do with the Uterus. Of course, women experience a much 
greater loss of Essence during the period and birth. But more, in another interesting article of mine!!!

\section{One Shiatsu session}

From my personal experience, I can say the following: Your Zen Shiatsu practitioner

i. Initially on your first session, will receive a not so brief medical history.

ii. While you are answering the medical history, he/she will be watching over you many small things, from the way you answer the questions, and your movements to your behavior from the moment you entered the place/spa! This, rather not out of rude curiosity, but so that he/she will diagnose by observation the dominant of the five elements and your Yin Yang characteristics, that we discussed above.Also, to record some of your external facial features mostly. He/she may need to check your tongue and take your pulse by both hands.

In each session, he/she will first palpate your belly area to pull one diagnosis from the Hara (in Japanese the area around the navel).

iii. In your next session he/she will be better prepared and will come to some conclusions, according to the answers you gave to the medical history. He/she will have set a plan, a strategy that will march with during your next sessions.

iv. The session is set in a clean quiet simple comfortable Zen space, with low soft ambient music or even without music, in meditative atmosphere that travels you to another positive world.
In order a good session Zen Shiatsu to be performed, the therapist needs to have proper professional training and enough experience. Both require many years, without this does to mean that a new Shiatsu therapist will not be able to relax you.

Sources/Bibliography: In order to write the above article I needed to use the following books from my study years:

i. The Foundations of Chinese Medicine, Giovanni Maciocia.

ii. Acupressure for common ailments, Chris Jarmie \& John Tindall.

iii. Shiatsu, The complete guide, Chris Jarmie \& Gabriel Mojay \& Ilaira Bouratinou.

iv. A practical Guide to Acu-points, Chris Jarmie \& Ilaira Bouratinou.

v. Meridian Exercises, The Oriental way to health and vitality, Shizuto Masunaga.

vi. Personal notes and client files from my professional experience up today.

\section{Acknowledgments}

None.

\section{Conflicts of interest}

Author declares there are no conflicts of interest.

\section{Funding}

None. 